\title{
Exploration of Direct-Ink-Write 3D Printing in Space: Droplet Dynamics and Patterns Formation in Microgravity
}

\author{
Weibin $\mathrm{Li}^{1,2}$. Ding Lan ${ }^{1,2} \cdot$ Yuren Wang ${ }^{1,2}$ \\ Received: 23 April 2020 / Revised: 8 July 2020 / Accepted: 13 July 2020 \\ (C) Springer Nature B.V. 2020
}

\begin{abstract}
As a simple, fast and effective 3D printing method, direct-ink-writing (DIW) has potential applications in repairing the circuit board in orbit, printing the wearable devices for the astronaut, and producing the solar cells for the energy supply in space. To expand the DIW technology to space, we designed the colloidal material box (CMB) as the prototype printer of DIW and verified its applicability in the Chinese SJ-10 satellite. The colloidal suspensions was adopted as a diluted ink model to investigate two key processes of DIW under microgravity environment: manipulation of the droplet and formation of the patterns. We have showed the dynamics of the droplet, which would determine the size of the features, could be controlled through tuning the wettability of the needles and the solid surface. Compared to the ground, the "coffee ring" effect was weakened for the drying patterns because of strong interfacial effect under weightless conditions. We have found that fast evaporation could assist for fabricating more uniform and ordered structures.
\end{abstract}

Keywords 3D printing $\cdot$ Direct-ink-write $\cdot$ Colloidal self-assembly $\cdot$ Droplet evaporation $\cdot$ Deposition pattern

\section{Introduction}

There are strong application requirements of the micro-nano structures in space, such as printed circuits (Jiang et al. 2018), sensors (Huang et al. 2011; Yang et al. 2020), solar cells (Bag et al. 2016), and tissue engineering (Zhu et al. 2016), which can used for spacecraft electronics (Werkheiser 2014), astronaut health monitoring (Hill 2019) and energy supply (Leake et al. 2016), respectively. Suppose that these structures and components can be fabricated directly in space, it will save a huge cost of space transportation and bring great convenience of replacement and maintenance in-orbit (Cowley et al. 2019; Fateri et al. 2018, 2019; Sacco and Moon 2019; Wang et al. 2020). However, the traditional "top-down" fabrication methods such as photoetching for chips are difficult to expand

Yuren Wang

yurenwang@imech.ac.cn

Ding Lan

landing@imech.ac.cn

1 National Microgravity Laboratory, Institute of Mechanics, Chinese Academy of Sciences, Beijing 100190, China

2 School of Engineering Science, University of Chinese Academy of Sciences, Beijing 100049, China in space for its expensive costs, complicated process and enormous consumption (Xiao 2001).

Compared to traditional manufacturing techniques, the three-dimensional (3D) printing has been proved to be an effective manufacturing technology that employ digitally controlled layer-by-layer deposition schemes to realize controllable composition and produce complex components and architectures (Gebhardt 2011; Guo and Leu 2013; Lipson and Kurman 2013; Wong and Hernandez 2012), therefore, it has been rapidly extended in the field of space manufacturing. Multiple 3D printing technologies on the basis of various materials (polymer, metal, ceramic, composite, etc.) was tested in space microgravity environment (Prater et al. 2017, 2019; Zocca et al. 2019). As a simple, fast and effective 3D printing method, the direct ink writing (DIW) technology can print a multitude of materials and construct arbitrary 3D structures through programmatically depositing fluid droplets on substrate surfaces (Lewis 2006; Lewis and Gratson 2004). Based on extensive utilizations for electronics, optics, and displays under terrestrial condition (Cox et al. 2001; Gao et al. 2012; Hon et al. 2008; Park et al. 2007; Zhang et al. 2012), DIW has tremendous potential application prospects in space if it can accommodate the extreme environments of space especially weightlessness. 
Both the fluid dynamics and the evaporation kinetics of ink droplets play important roles in defining the printing structures deposited by DIW (Lewis and Gratson 2004), therefore, it is necessary to clarify the gravitational effects on these two physical processes under the condition of weightlessness. Firstly, the inks should be injected or squeezed through a needle successfully. In microgravity environment, gravitational effects are negligibly small and surface tension dominates liquid behavior, thus the liquid tends to climb along the wetting surface or float away without the downward force of gravity, which increases the difficulties in droplet injection and manipulation (Dreyer et al. 1994; Meseguer et al. 2014; Stange et al. 2003). Secondly, the inks will solidify on solid substrate either by liquid evaporation or temperature-induced phase change. When the inks is injected and forms a sessile droplet, it always forms specific patterns such as the common "coffee ring" (Deegan et al. 1997) under the coupling physical effects after evaporation in normal gravity. However, the printing patterns will be altered for vanishing of gravitational sedimentation and buoyancy convection in microgravity. The difficulty in controlling evaporation kinetics limited their applicability to forming uniform and ordered structures in space. Therefore, studying the droplet manipulation and patterns formation in microgravity is the essential step of achieving DIW in space.

In this work, we have adopted the colloidal suspension as a diluted ink model to study the manipulation of the droplet and the formation of the drying patterns in space. We have studied the fluid dynamics of droplet injection, spreading and wetting on solid surface, and further revealed the self-assembly behavior of colloidal particles and formation mechanisms of the printed patterns under microgravity. All experiments were conducted in the colloidal material box (CMB) aboard the Chinese SJ-10 satellite (Hu et al. 2014).

\section{Experimental Payload}

The CMB is a subsystem and payload of Chinese SJ-10 satellite, as shown in Fig. 1. It is composed of four units: (i) a liquid injection and management unit for uniform dispersion and injection of colloidal suspensions; (ii) a displacement stage for motion and heating of the colloidal droplet; (iii) an optical observation unit for observation of droplet profiles and drying patterns; (iv) a drive control unit for automatic control of the experimental procedures in space. The detailed structures and functions of the $\mathrm{CMB}$ can be referred to our previous works ( $\mathrm{Li}$ et al. 2016a, b). This paper mainly introduces the experimental principle and operation processes as follows: Before the experiment, the polystyrene (PS) colloidal suspension (particle size: $3 \mu \mathrm{m}$, concentration: $0.1 \sim 0.15 \mathrm{wt} \%$, Duke (5200)) in the reservoir was mixed thoroughly about $15 \mathrm{~min}$ under ultrasonic stirring, which ensures the uniform concentration of the colloidal suspensions and prevents the needle blocking. Subsequently, the quartz substrate placed on the displacement stage was precisely positioned under the needle, and the substrate was preheated for above $40-50{ }^{\circ} \mathrm{C}$ to accelerate the evaporation. When the experiments began, the colloidal suspension was injected from the printing needle onto the heated substrate and formed sessile droplets, then the colloidal particles self-assembled relying on the interfaces as with droplet evaporation. The injection and wetting process of the droplet could be observed by a macroscopic CCD camera, and particles self-assembling and final depositions could be recorded by a microscope, as shown in Fig. 2. The specific patterns could be obtained theoretically through changing the positions of the displacement stage and repeating the processes of injection and evaporation. The internal structure and functional units of the $\mathrm{CMB}$ can be seen in Fig. 1.

To verify the applicability of the DIW technology in space, we had conducted several experiments of droplet manipulation and patterns formation under microgravity conditions. The real-time images and videos of droplet injection and evaporation from a macro camera, and colloidal particles assembly and final patterns from a microscope were simultaneously filmed. Manipulation and evaporation of different initial volumes of colloidal droplets $(20-160 \mu \mathrm{L})$ were tested and investigated. In addition, deposition of the colloidal particles relying on interfaces of solid surface was studied in terrestrial gravity and microgravity, respectively.

\section{Droplet Dynamics of Injection and Wetting}

The flow behavior during printing is relied on the rheological properties of the colloidal materials, especially for the extrusion-based systems. For droplet-based printing system, most inks are Newtonian fluids. Therefore, the surface tension of inks are of vital importance to the printing. In fact, injection and spreading of the diluted ink droplet on solid surface were dominated by the coupling effects of surface tension and gravity. The interrelation between gravity and surface tension can be described by the dimensionless Bond number, $B o=\rho g L^{2}$ $/ \gamma$, where $L$ is the characteristic length scale, $\gamma$ is the surface tension, $\rho$ is the liquid density, and $\mathrm{g}$ is the gravity constant (Diana et al. 2012). In microgravity environment, $g=10^{-3} \mathrm{~g}_{0}$, thus $\mathrm{Bo}<<1$. Therefore, the effects due to gravity are negligible, and the liquid shapes from injection is controlled by surface tension. Thus the liquid remains more unstable without the downward restriction of gravity in space, which increases the difficulty of droplet control and management in orbit. We found the injecting liquid was easily to climb along 
Fig. 1 Simulated assembly drawing of the CMB

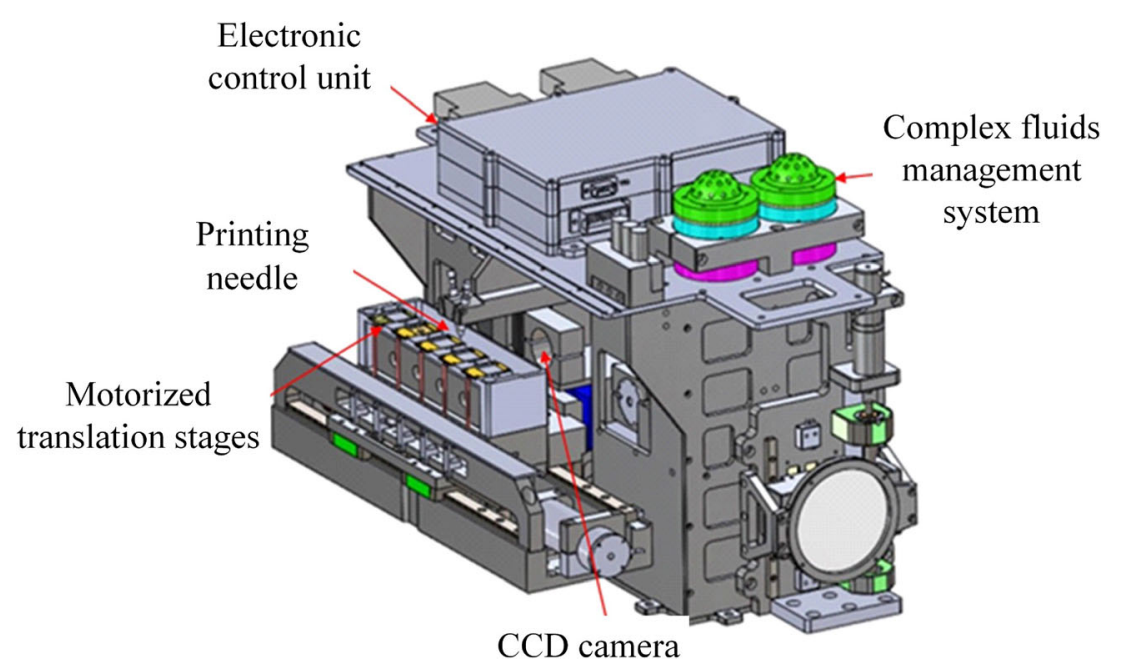

the needles even for the large volume of droplet, which might result in the failure of injection in microgravity, as shown in Fig. 3b. Because of the dominant interfacial tensions, the printing process of injection and spreading can be manipulated through tuning the wettability of the needles and the solid surface, and the surface tension of the colloidal inks.

The water-based PS colloidal suspensions were injected from a miniature electromagnetic pump (LPVA, Lee Co.) at a rate of $11.42 \mu \mathrm{L} / \mathrm{s}$. The needles (062 MINSTAC, Lee Co.), whose inner and outer diameters were 0.1 and $1.3 \mathrm{~mm}$, respectively, were treated as hydrophobic to repel the water solution from climbing. Two hydrophobic needles were adopted as the printing head, and the hydrophilic quartz glass was chosen as the solid substrate. In the injection process, the droplet first stayed at the pinpoint of the needle. As the liquid volume increased, the droplet would contact the hydrophilic surface and then spread out rapidly, it finally formed the sessile droplet with balanced contact angle and specific contact area. Sizes

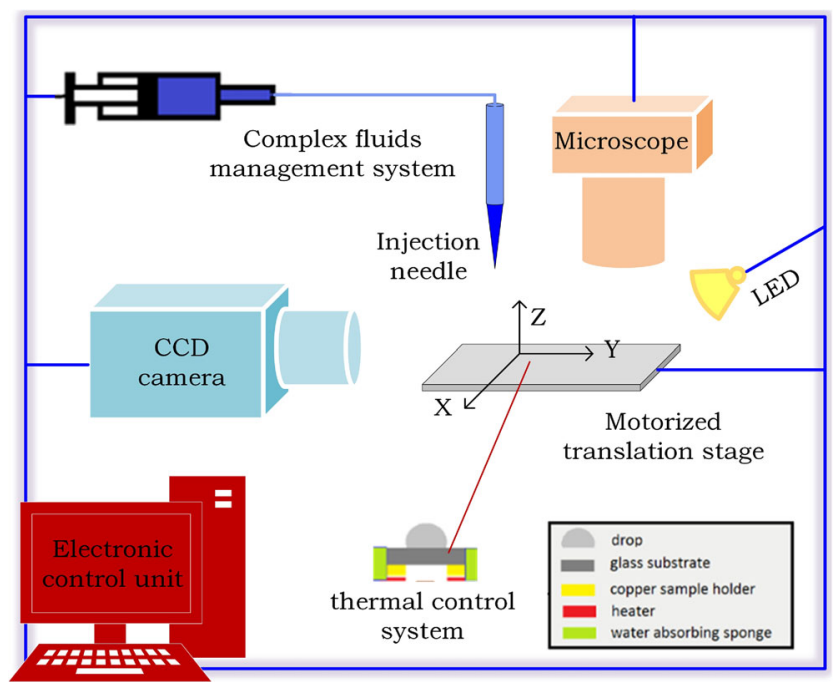

Fig. 2 Working principle diagram of the CMB of the final pattern is probably fixed already at this spreading stage. It is worth mentioning that the spreading process can be controlled by the roughness and heterogeneity of the solid surface, which implies that the printing patterns can be regulated through modifying the surface properties. In the experiments, a specific hydrophobic circle (diameter: $\sim 5 \mathrm{~mm}$ ) was designed to restrain the spreading of the colloidal droplet because the aqueous suspension tended to wet the hydrophilic region but dewet the hydrophobic region, as shown in Fig. 3 a. The pinned droplet would evaporate as constant contact radius (CCR) mode (Fig. 3c), therefore, we finally obtained patterns with fixed areas and sizes.

\section{Colloidal Self-assembly and Patterns Formation}

The morphology of the dried patterns that directly reflects the printing quality is highly related to the evaporation process. So it is necessary to investigate the kinetics of evaporation and the dynamics of particles deposition. Previous studies have shown that the deposition patterns are determined by complex interactions of the micro-flow (including capillary flow and Marangoni flow) (Deegan et al. 1997, 2000; Hu and Larson 2005, 2006), contact line behavior (pinning and de-pinning) (Li et al. 2013; Orejon et al. 2011), interfacial effects (such as liquid-air interface capture, liquid-solid interaction) (Bhardwaj et al. 2010; Li et al. 2016a, b; Nguyen et al. 2017), and solutes properties (sizes, shapes, etc.) (Li et al. 2019; Yunker et al. 2011). Therefore, the patterns can be regulated through controlling these multiple physical effects. Despite great process, the gravitational effects on the evaporative convection and deposition still lacks full understanding. Here, we have further investigated the formation mechanism of the drying patterns in microgravity to verify the feasibility of DIW in space. 
Fig. 3 a Injection of the colloidal droplet. b Failed injection due to the droplet climbing along the needles. (c) Evolution of the droplet shape as with liquid evaporation, the scale bar is $5 \mathrm{~mm}$

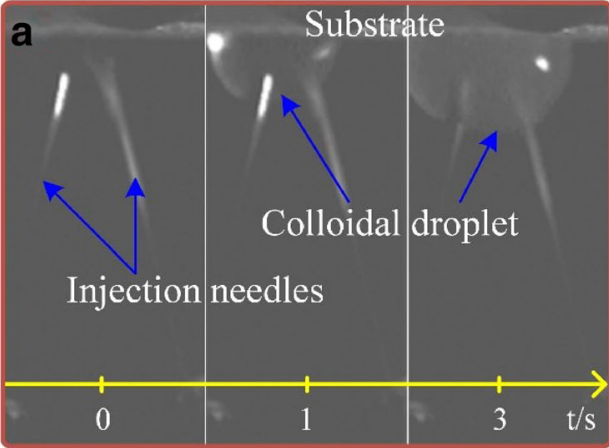

Successful injection

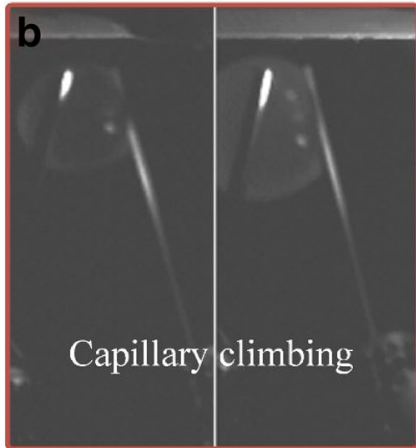

Failed injection

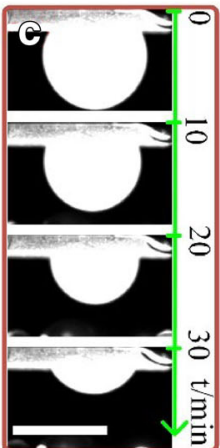

Evaporation
Experimental results show the interfacial effect dominate the deposition of colloidal particles under microgravity environment for the absent of gravity sedimentation and buoyancy convection. Therefore, we focus on the micro-flow and selfassembly dynamics relying on the interfaces of a droplet, including the liquid-air interface, the liquid-solid interface, and the triple interfaces of liquid, solid and air. Figure 4 shows the dynamic deposition of the colloidal particles and the eventual patterns under microgravity condition. When the contact angle was large, most particles were easily to be captured by the shrinking liquid-air interface and formed islands and pieces of monolayer structures because the gravitational sedimentation was negligible. When the contact angle was small, few colloidal particles were pushed towards the contact line under the outward capillary flow and finally formed the weakened ring- like structure, as shown in Fig. 4a. The capillary flow was resulted from the non-uniform evaporation flux between the edge and the center, which was not influenced by gravity (Deegan et al. 1997). These structures on the liquid-air interface, strongly affected by the Marangoni flow (as seen in the black region in Fig. 4b), would eventually transfer to the solid surface after evaporation and rearranged into monolayer and multilayer structures under the action of liquid film dewetting, as shown in Fig. 4c \& e. Figure $4 \mathrm{f}$ shows that the monolayers has stable hexagonal close-packed (hcp) structure containing voids. Therefore, we can obtain more uniform pattern in microgravity because of the "weakened coffee ring".

To further understand the effects of gravity and heating on the patterns, we had conducted experiments of droplet evaporation with an increased temperature $\left(50^{\circ} \mathrm{C}\right)$ under terrestrial
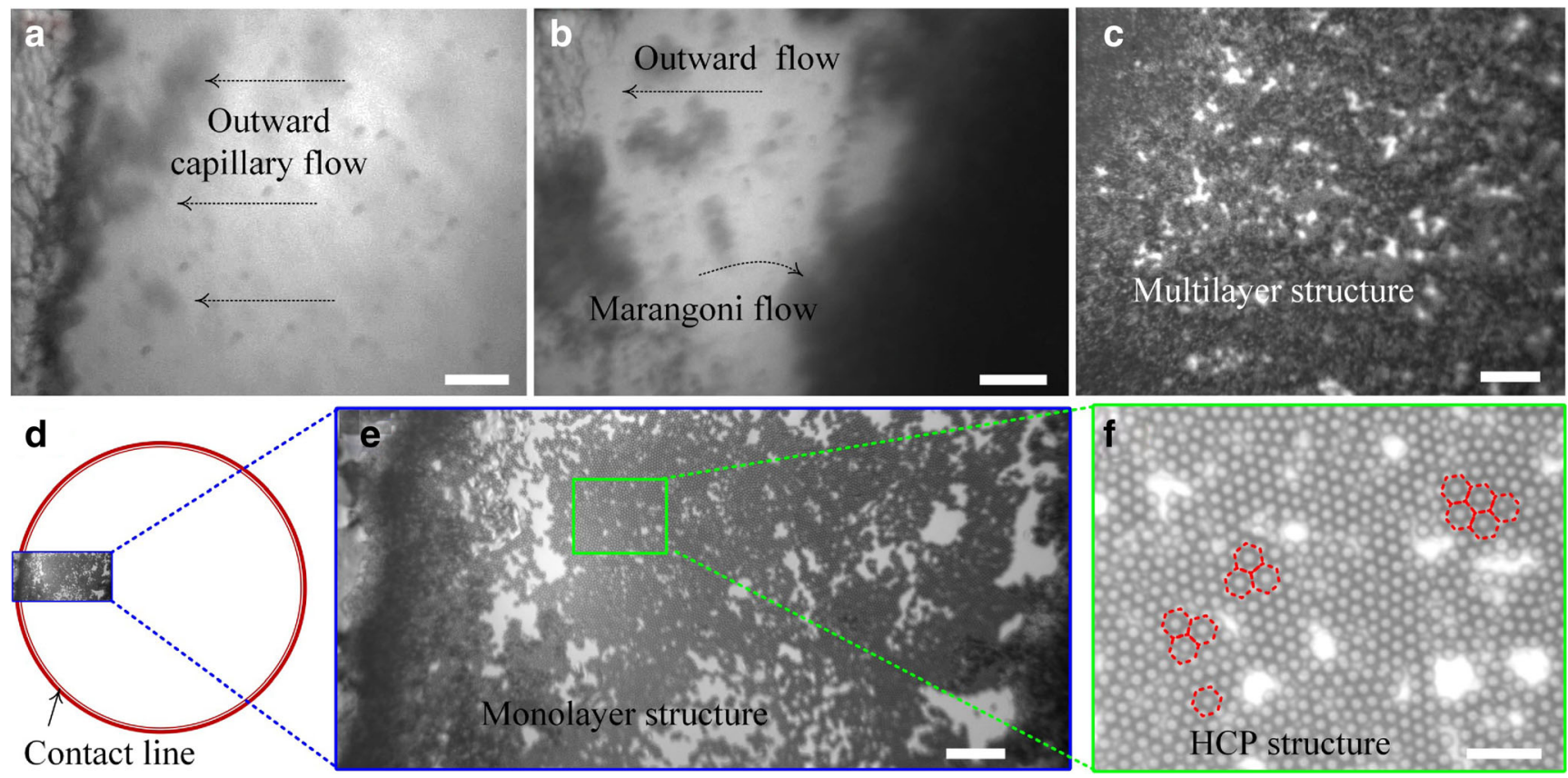

Fig. 4 Patterns formation from evaporating droplets on a heating substrate $\left(40{ }^{\circ} \mathrm{C}\right)$ under microgravity. a Particles was carried toward the contact line under the outward capillary flow. b The self-assembled monolayers at the liquid-air interface moved under the combined action of the Marangoni flow and the capillary flow. c Multilayer structures in the center of the ring. d-e The outer ring-like structure and inner monolayer structure with particle-free region. $\mathbf{f}$ The local region of $\mathbf{e}$ has hcp structure. The bar of (a), (b), (c), (e), (f) is $30 \mu \mathrm{m}, 50 \mu \mathrm{m}$, $60 \mu \mathrm{m}, 60 \mu \mathrm{m}$, and $15 \mu \mathrm{m}$, respectively 

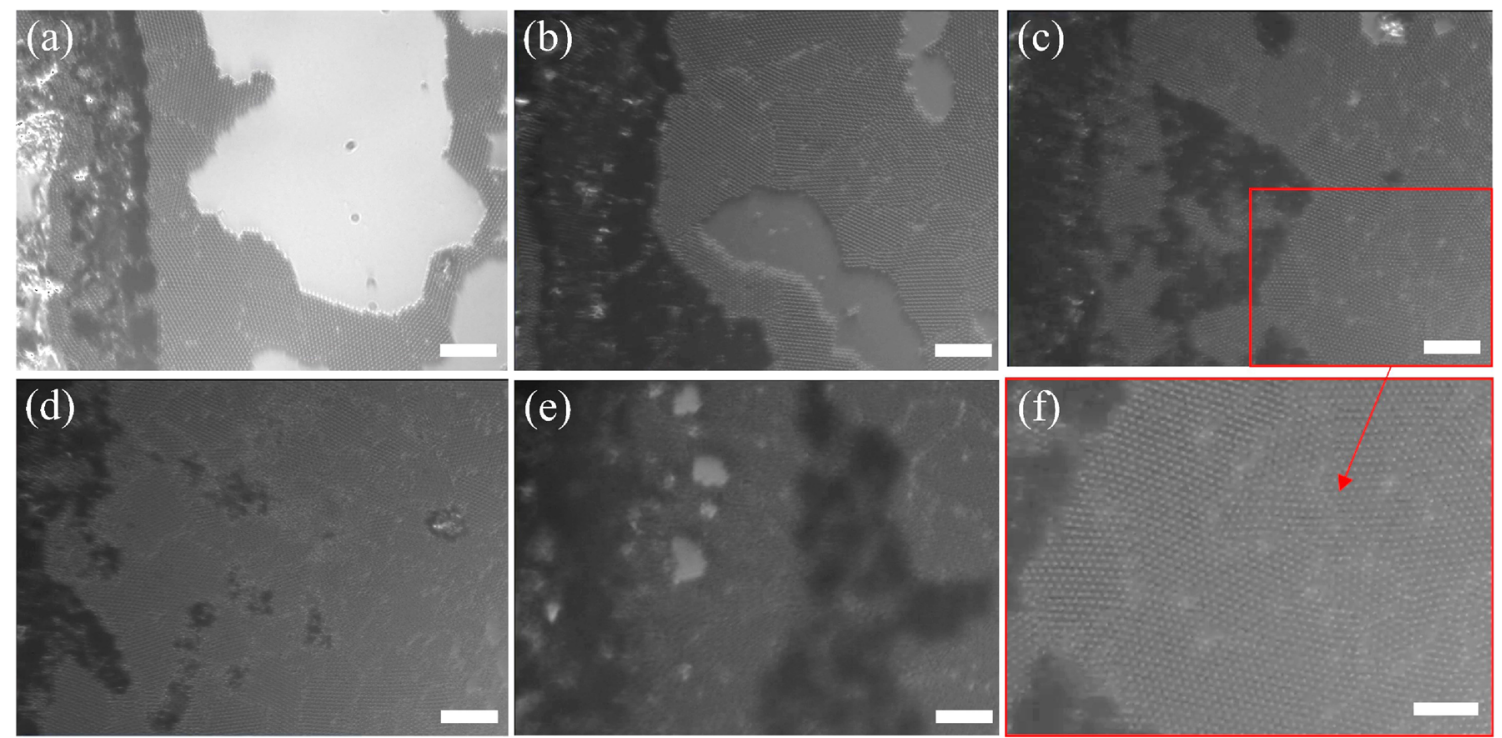

Fig. 5 Final deposition patterns for colloidal droplets with different initial volumes (a) $30 \mu \mathrm{L}$ (b) $40 \mu \mathrm{L}$ (c) $50 \mu \mathrm{L}$ (d) $60 \mu \mathrm{L}$ (e) $70 \mu \mathrm{L}$ on a heating substrate $\left(50{ }^{\circ} \mathrm{C}\right)$ under normal gravity, the concentration is $0.14 \mathrm{wt} \%$; (f)

gravity. The droplet evaporated as CCR mode. Figure 5 shows the deposition patterns for colloidal droplets with different initial volumes $(30,40,50,60,70 \mu \mathrm{L} ; 0.14 \mathrm{wt} \%)$. It formed the common ring-like pattern with multilayer structures at the edge of the droplet. This inner structures of the ring show ordered arrays of sub-monolayer, monolayer and bilayer by increasing the initial volumes, as shown in Fig. 5a-e. Figure $5 \mathrm{f}$ is an enlarged image in red square of Fig. $5 \mathrm{c}$, which shows that the colloidal particles has a hop structure. Unlike the ring-like structure caused by capillary flow, the ordered hcp structures in the center could be attributed to interface capture effect. Compared with microgravity environment, there was a stronger "coffee ring" effect in normal gravity because of more particles remaining in the droplet at the last stage of evaporation due to the particles sedimentation and the weak interfacial effects. Liquid-air interface can promote the formation of monolayer ordered structures, because the particles captured by the liquid-air interface always experience the capillary attraction initiated by interfacial deformation. Therefore, substrate heating not only shortened the drying time, but also induced formation of the ordered structures through fast evaporation. This provides an excellent opportunity to print periodic structures.

\section{Conclusions}

In this work, we have designed the CMB as the prototype printer to investigate the applicability of DIW technology in space. Both the fluid dynamics and the patterns formation are the deciding factors in the success of DIW. Therefore, the colloidal suspensions was adopted as a diluted ink model is an enlarged image in red square of (c). The scale bar for (a)-(e) is $30 \mu \mathrm{m}$ and for (f) is $15 \mu \mathrm{m}$

system to investigate the droplet manipulation and evaporation-induced deposition of colloidal particles in microgravity environment. The dynamics of droplet injection and spreading was firstly analyzed, which shows that the droplet could be manipulated through tuning the wettability of the needle and the solid surface. The self-assembly behavior of the colloidal particles and formation mechanisms of the printed patterns was further revealed, which indicated that the "coffee ring" effect was weakened because of the strong interfacial effect in microgravity. Combined with fast evaporation through substrate heating, it could be able to fabricate more uniform and ordered structures.

Acknowledgements The authors gratefully acknowledge financial support from National Natural Science Foundation of China (Grant No. 11902321 and U1738118) and the Strategic Priority Research Program on Space Science, the Chinese Academy of Sciences (A) (Grant Nos. XDA04020202 and XDA04020406).

\section{References}

Bag, M., Jiang, Z., Renna, L.A., Jeong, S.P., Rotello, V.M., Venkataraman, D.: Rapid combinatorial screening of inkjet-printed alkyl-ammonium cations in perovskite solar cells. Mater. Lett. 164, 472-475 (2016)

Bhardwaj, R., Fang, X., Somasundaran, P., Attinger, D.: Self-assembly of colloidal particles from evaporating droplets: role of DLVO interactions and proposition of a phase diagram. Langmuir 26(11), 7833$7842(2010)$

Cowley, A., Perrin, J., Meurisse, A., Micallef, A., Fateri, M., Rinaldo, L., Bamsey, N., Sperl, M.: Effects of variable gravity conditions on additive manufacture by fused filament fabrication using polylactic acid thermoplastic filament. Addit. Manuf. 28, 814-820 (2019)

Cox, W.R., Chen, T., Hayes, D.J.: Micro-optics fabrication by ink-jet printers. Opt. Photonics News 12(6), 32-35 (2001) 
Deegan, R.D., Bakajin, O., Dupont, T.F., Huber, G., Nagel, S.R., Witten, T.A.: Capillary flow as the cause of ring stains from dried liquid drops. Nature 389(6653), 827-829 (1997)

Deegan, R.D., Bakajin, O., Dupont, T.F., Huber, G., Nagel, S.R., Witten, T.A.: Contact line deposits in an evaporating drop. Phys. Rev. E 62, 756-765 (2000)

Diana, A., Castillo, M., Brutin, D., Steinberg, T.: Sessile Drop Wettability in Normal and Reduced Gravity. Microgravity Sci. Technol. 24(3), 195-202 (2012)

Dreyer, M., Delgado, A., Path, H.-J.: Capillary Rise of Liquid between Parallel Plates under Microgravity. J. Colloid Interf. Sci. 163(1), 158-168 (1994)

Fateri, M., Kaouk, A., Cowley, A., Siarov, S., Palou, M.V., González, F.G., Marchant, R., Cristoforetti, S., Sperl, M.: Feasibility study on additive manufacturing of recyclable objects for space applications. Addit. Manuf. 24, 400-404 (2018)

Fateri, M., Pitikaris, S., Sperl, M.: Investigation on wetting and melting behavior of lunar regolith simulant for additive manufacturing application. Microgravity Sci. Technol. 31(2), 161-167 (2019)

Gao, Y.X., Li, H.Y., Liu, J.: Direct writing of flexible electronics through room temperature liquid metal ink. PLoS One 7(9), e45485 (2012)

Gebhardt, A.: Understanding additive manufacturing: rapid prototyping, rapid tooling, rapid manufacturing. Hanser, Munich (2011)

Guo, N., Leu, M.C.: Additive manufacturing: technology, applications and research needs. Front. Mech. Eng. 8(3), 215-243 (2013)

Hill, C.: Flexible Sensor Development for Astronaut Crew Health Monitoring. (2019). https://ntrs.nasa.gov/archive/nasa/casi.ntrs. nasa.gov/20200000034.pdf. [online]

Hon, K., Li, L., Hutchings, I.: Direct writing technology—Advances and developments. CIRP Ann. 57(2), 601-620 (2008)

$\mathrm{Hu}, \mathrm{H}$., Larson, R.G.: Analysis of the effects of Marangoni stresses on the microflow in an evaporating sessile droplet. Langmuir 21(9), 39723980 (2005)

Hu, H., Larson, R.G.: Marangoni effect reverses coffee-ring depositions. J. Phys. Chem. B 110(14), 7090-7094 (2006)

Hu, W.R., Zhao, J.F., Long, M., Zhang, X.W., Liu, Q.S., Hou, M.Y., Kang, Q., Wang, Y.R., Xu, S.H., Kong, W.J., Zhang, H., Wang, S.F., Sun, Y.Q., Hang, H.Y., Huang, Y.P., Cai, W.M., Zhao, Y., Dai, J.W., Zheng, H.Q., Duan, E.K., Wang, J.F.: Space program SJ10 of microgravity research. Microgravity Sci. Technol. 26(3), 159169 (2014)

Huang, L., Huang, Y., Liang, J.J., Wan, X.J., Chen, Y.S.: Graphenebased conducting inks for direct inkjet printing of flexible conductive patterns and their applications in electric circuits and chemical sensors. Nano Res. 4(7), 675-684 (2011)

Jiang, P., Ji, Z.Y., Zhang, X.Q., Liu, Z.L., Wang, X.L.: Recent advances in direct ink writing of electronic components and functional devices. Prog. Addit. Manuf. 3(1-2), 65-86 (2018)

Leake, S., McGuire, T., Parsons, M., Hirsch, M.P., Straub, J.: Powering an in-space 3D printer using solar light energy. Proceedings of the SPIE Defense + Comercial Sensing Conference: (2016)

Lewis, J.A.: Direct Ink Writing of 3D Functional Materials. Adv. Fun. Mater. 16(17), 2193-2204 (2006)

Lewis, J.A., Gratson, G.M.: Direct writing in three dimensions. Mater. Today 7(7-8), 32-39 (2004)

Li, Y.F., Sheng, Y.J., Tsao, H.K.: Evaporation stains: suppressing the coffee-ring effect by contact angle hysteresis. Langmuir 29(25), 7802-7811 (2013)

Li, W.B., Lan, D., Sun, Z.B., Geng, B.M., Wang, X.Q., Tian, W.Q., Zhai, G.J., Wang, Y.R.: Colloidal Material Box: In-situ Observations of Colloidal Self-Assembly and Liquid Crystal Phase Transitions in Microgravity. Microgravity Sci. Technol. 28(2), 179-188 (2016a)

Li, Y.N., Yang, Q., Li, M.Z., Song, Y.L.: Rate-dependent interface capture beyond the coffee-ring effect. Sci. Rep. 6, 24628 (2016b)
Li, W.B., Ji, W.J., Sun, H.H., Lan, D., Wang, Y.R.: Pattern Formation in Drying Sessile and Pendant Droplet: Interactions of Gravity Settling, Interface Shrinkage, and Capillary Flow. Langmuir 35(1), 113-119 (2019)

Lipson, H., Kurman, M.: Fabricated: The new world of 3D printing. Wiley, Hoboken (2013)

Meseguer, J., Sanz-Andrés, A., Pérez-Grande, I., Pindado, S., Franchini, S., Alonso, G.: Surface tension and microgravity. Eur. J. Phys. 35(5), 055010 (2014)

Nguyen, T.A.H., Biggs, S.R., Nguyen, A.V.: Manipulating colloidal residue deposit from drying droplets: Air/liquid interface capture competes with coffee-ring effect. Che. Eng. Sci. 167, 78-87 (2017)

Orejon, D., Sefiane, K., Shanahan, M.E.: Stick-slip of evaporating droplets: substrate hydrophobicity and nanoparticle concentration. Langmuir 27(21), 12834-12843 (2011)

Park, B.K., Kim, D., Jeong, S., Moon, J., Kim, J.S.: Direct writing of copper conductive patterns by ink-jet printing. Thin Solid Films 515(19), 7706-7711 (2007)

Prater, T., Bean, Q., Werkheiser, N., Grguel, R., Beshears, R., Rolin, T., Huff, T., Ryan, R., Ledbetter, F., Ordonez, E.: Analysis of specimens from phase I of the 3D printing in Zero G technology demonstration mission. Rapid Prototyp. J. 23(6), 1212-1225 (2017)

Prater, T., Werkheiser, N., Ledbetter, F., Timucin, D., Wheeler, K., Snyder, M.: 3D Printing in Zero G Technology Demonstration Mission: complete experimental results and summary of related material modeling efforts. Int. J. Adv. Manuf. Technol. 101, 391-417 (2019)

Sacco, E., Moon, S.K.: Additive manufacturing for space: status and promises. Int. J. Adv. Manuf. Technol. 105(10), 4123-4146 (2019)

Stange, M., Dreyer, M.E., Rath, H.J.: Capillary driven flow in circular cylindrical tubes. Phys. Fluids 15(9), 2587-2601 (2003)

Wang, G., Zhao, W., Liu, Y.F., Cheng, T.J.: Review of space manufacturing technique and developments (in Chinese). Sci Sin-Phys Mech Astron. 50(4), 047006 (2020)

Werkheiser, N.: Overview of nasa initiatives in 3D printing and additive manufacturing. (2014). https://ntrs.nasa.gov/archive/nasa/casi.ntrs. nasa.gov/20150002612.pdf. [online]

Wong, K.V., Hernandez, A.: A review of additive manufacturing. ISRN Mech. Eng. 2012, 1-10 (2012)

Xiao, H.: Introduction to semiconductor manufacturing technology. Prentice-Hall Inc., New Jersey (2001)

Yang, W.W., Zhao, W., Li, Q.S., Li, H., Wang, Y.L., Li, Y.X., Wang, G.: Fabrication of Smart Components by 3D Printing and LaserScribing Technologies. ACS Appl. Mater. Interfaces 12(3), 3928 3935 (2020)

Yunker, P.J., Still, T., Lohr, M.A., Yodh, A.G.: Suppression of the coffee-ring effect by shape-dependent capillary interactions. Nature 476(7360), 308-311 (2011)

Zhang, Q., Zheng, Y., Liu, J.: Direct writing of electronics based on alloy and metal (DREAM) ink: A newly emerging area and its impact on energy. environment and health sciences. Front. Energy 6(4), 311$340(2012)$

Zhu, W., Ma, X.Y., Gou, M.L., Mei, D.Q., Zhang, K., Chen, S.C.: 3D printing of functional biomaterials for tissue engineering. Curr. Opin. Biotechnol. 40, 103-112 (2016)

Zocca, A., Lüchtenborg, J., Mühler, T., Wilbig, J., Mohr, G., Villatte, T., Léonard, F., Nolze, G., Sparenberg, M., Melcher, J., Hilgenberg, K., Günster, J.: Adv. Mater. Technol. 4(10), 1900506 (2019)

Publisher's Note Springer Nature remains neutral with regard to jurisdictional claims in published maps and institutional affiliations. 